\title{
The treatment of sarcoidosis
}

\author{
HAROLD L. ISRAEL
}

\section{Sarcoidosis Clinic, Thomas Jefferson University School of Medicine, Philadelphia}

\begin{abstract}
Summary
A discouraging conclusion to many years of study of the natural history of sarcoidosis is the realization that the proper word for the course of sarcoidosis is 'unpredictable'. An impressive lesson of a controlled study was the frequency with which unexpected and dramatic improvement occurred in the placebo group. Although patients with pulmonary infiltration, uveitis and cutaneous sarcoid have a worse prognosis than the asymptomatic patient with hilar adenopathy, many patients in the former category will recover and some in the latter will go on to death.

A trial of prednisone therapy in a small number of patients with disease restricted to the mediastinal and hilar nodes (Stage I) suggests that prednisone treatment in this form of sarcoidosis may minimize dissemination and progression. An extended trial in Stage I sarcoidosis appears warranted. In more disseminated forms of the disease, the effects of corticosteroids seem to be merely palliative: there is no persuasive evidence that their use in pulmonary sarcoidosis averts fibrosis.

Occasionally corticosteroid therapy is contraindicated, poorly tolerated, or ineffective. In such circumstances chlorambucil or methotrexate may be given a trial. It is clear that both drugs exert an antiinflammatory action similar to that of the corticosteroids; in a few instances these agents appear to surpass corticosteroids in effectiveness.
\end{abstract}

Corticosteroid treatment was introduced shortly after sarcoidosis became commonly recognized. Few observations are as a result available regarding the natural history of the disease. The therapeutic effects of corticosteroids are so well established in ocular, myocardial, CNS and endocrine forms of sarcoidosis that their use cannot be withheld for purposes of therapeutic trials. Prednisone therapy, in most series, has been given to approximately a third of patients indicating that clinicians in all countries usually find that two-thirds of patients have benign disease not requiring treatment.

The course and prognosis of sarcoidosis appear to be influenced by many factors. Progressive and fatal disease is somewhat more frequent in American studies than in European ones, in part because of the predominance of Negro patients in the former. The differences are merely ones of degree, however: advanced pulmonary fibrosis is by no means infrequent among white American patients and the lesser likelihood of a fatal end in this race may largely reflect educational and economic advantages which result in earlier and more constant medical care. Even greater than these effects, however, is the influence of selection. Physicians chiefly involved in hospital practice, whether in Norway, Great Britain or the United States, report a high frequency of progressive and malignant disease while physicians dealing with asymptomatic patients whose sarcoidosis was detected in radiographic surveys will describe a disease that is usually benign.

The one major difference in behaviour of sarcoidosis between the United States and Europe, is that of the course of Stage I disease. Most English and Scandinavian studies indicate an almost invariably benign course in patients free of pulmonary infiltration at the time of diagnosis, although Scadding notes that a third of his patients with hilar adenopathy developed pulmonary infiltration under observation. This experience resembles that of American investigators where the prognosis in Stage I is not significantly better than in Stages II and III. In a recent Philadelphia study, improvement was observed in $63 \%$ of patients who had entered with hilar adenopathy alone, and $52 \%$ of those who had had pulmonary invasion when first observed. But although asymptomatic patients detected in routine surveys have a greater likelihood of quick recovery than patients ill with breathlessness, uveitis, or other extrapulmonary manifestations, the prognosis in the individual case is unpredictable. Some patients discovered with Stage I disease clear completely, others have massive adenopathy for years, and others develop pulmonary infiltration which in turn may be transient or progressive. Spontaneous clearing may occur in a few months, or only after several years. It is a question whether the most remarkable feature of sarcoidosis is the spontaneous clearing of dense infiltrates or the insidious development of diffuse fibrosis and cysts. 
Pulmonary physiologists have tended to study symptomatic patients and have been impressed by the frequency of respiratory impairment even in patients with minimal radiologic evidence of damage. Physicians with this experience are likely to urge the wide use of steroid therapy in the hope of averting fibrosis. Physicians in out-patient clinics see patients whose course is as a rule favourable, so that treatment of all patients with hazardous drugs appears unjustifiable. Physicians whose experience encompasses both groups are likely to conclude that corticosteroids suppress the manifestations of sarcoidosis without altering the eventual outcome (Scadding, 1967; Israel, Sones \& Harrell, 1954). Although the immediate effects of corticosteroids on the lesions of sarcoidosis, as demonstrated by pathologic (Sones et al., 1951), physiologic (Sharma, Colp \& Williams, 1966) and clinical studies (Hoyle, 1967) are quite consistent and often striking, the long term effects are less impressive. It might be anticipated that the symptomatic effects of prednisone would make this drug a popular one with patients. On the contrary, many patients are dismayed by enormous weight gain; this is a frequent cause of cessation of treatment, in women especially.

Trial-and-error experience has taught some generally accepted lessons:

Indications for treatment: the readily demonstrable value of steroids for ocular, myocardial and neural involvement and for hypercalcaemia makes their use essential in these circumstances. It has generally been considered obvious that therapy is not indicated for the asymptomatic patient whose only abnormality is hilar adenopathy.

Difference in practice actually exists chiefly in the case of patients with pulmonary infiltrates, few or no symptoms and minimal functional impairment. Opinions differ sharply as to whether corticosteroid therapy should be used to reduce the inflammatory reaction observed on radiographic examination.

Dosage: Unless a patient is acutely ill there is no need for high doses of prednisone. Institution of therapy with daily dosage of $15 \mathrm{mg}$ is adequate for the majority of patients. If higher doses appear necessary to obtain the desired effects, alternate-day administration is a useful method of reducing adrenal gland suppression. Whatever level is used at the outset, the dosage is gradually reduced to the smallest level which maintains the improvement which has been obtained. One of the remarkable aspects of the prednisone therapy of sarcoidosis is the effect of small doses; in many cases, gradual reduction in daily dosage from 20 to $5 \mathrm{mg}$ has maintained clinical and radiologic improvement, while cessation of this minute maintenance dose has been followed by prompt relapse.

Duration of therapy: This is the aspect of treat- ment on which there is the greatest difference in practice. The rate of relapse after termination of corticosteroid therapy is distressingly high. The frequency of its occurrence has led investigators to advocate longer and longer treatment courses in the hope of averting relapse; Deenstra \& Van Ditmars (1968) have recommended a minimum of 3-years' treatment. A more common practice is to treat chronic cases for a minimum of 6 months. If relapse occurs, treatment is given for 1 year, and if relapse again occurs, a 2-year course of treatment is given. Relapse usually occurs from 1 to 4 months after steroids are stopped. Later relapse is rare. Recurrence after apparent complete clearing has been observed only in three instances in our experience, once in a man and twice in young women following delivery.

The difficulties of designing and carrying out a controlled trial of treatment in sarcoidosis are obvious. It is unjustifiable to give prednisone in large doses for 6 months or more to patients with a disease which has a better than even chance of improving spontaneously. Furthermore asymptomatic patients are not likely to remain under the intensive and prolonged supervision welcomed by the ill minority. Despite these obstacles a controlled trial carried out in Philadelphia for the past seven years has been instructive (Israel \& Beggs, 1969). Ninety patients with previously untreated active sarcoidosis were given $15 \mathrm{mg}$ of prednisone or placebo tablets daily for 3 months and seventy-nine completed the course of treatment and remained under observation for at least a year thereafter. By random allocation thirty-seven patients received prednisone and forty-two patients received inert tablets; the two groups proved to be similar in race, age and sex as well as in the stage of disease. An interesting demonstration that the sample of seventynine patients was one representative of American patients was provided by combining the treated and placebo groups. The percentages recovered, improved and worse were almost identical with those reported in large series of patients in Philadelphia (Sones \& Israel, 1960) and New York (Siltzbach, 1967).

The results of the trial may be summarized as follows:

Three month evaluation-Stage I: On completion of treatment $61 \%$ of eighteen patients in the steroid group and $35 \%$ of the seventeen controls showed overall improvement. Radiologic improvement occurred in $39 \%$ of those treated and $24 \%$ of the placebo group. One treated and one untreated patient had a normal chest film by the end of the 3 month trial; in neither group was X-ray progression noted.

Three month evaluation-Stages II and III: $53 \%$ of nineteen treated patients showed overall improve- 
ment and $42 \%$ were unchanged. In contrast, $17 \%$ of twenty-five controls improved while $83 \%$ were unchanged. This difference is significant $(P<0.05)$. Roentgenographic evaluation revealed $37 \%$ improvement in the steroid group and $16 \%$ improvement in controls. Roentgenographic appearances remained unchanged in eighteen controls and nine treated patients, and worsened in two controls.

Long term evaluation-Stage I: After a mean interval of observation of 3.3 years, $72 \%$ of treated patients and $47 \%$ of controls showed overall improvement. On roentgenographic evaluation improvement was noted in $78 \%$ of those treated and $47 \%$ of the controls. A normal chest film was achieved by seven treated patients. One treated patient and four controls showed radiographic progression of disease.

Long term evaluation-Stages II and III: After a mean interval of observation of 3.5 years, $47 \%$ of treated patients showed overall improvement as did $56 \%$ of the controls. $47 \%$ of treated patients showed roentgenographic improvement and $32 \%$ showed progression. $48 \%$ of controls were improved and $28 \%$ worse. Twelve patients achieved complete $\mathrm{X}$-ray remission, seven in the treated group and five controls.

A dosage schedule of $15 \mathrm{mg}$ of prednisone daily for 3 months was selected as a compromise between higher and more prolonged doses that might have produced more marked effects, and the consideration that higher doses involve a significantly increased hazard of complications and adrenal suppression. Such hazards could not be justified in a disease from which a majority of patients will recover without treatment. Despite the brevity of the treatment period, a surprising long-term advantage was noted in Stage I patients receiving prednisone. The difference between treated and control groups did not achieve statistical significance, but does not appear to be due to chance allocation. It has been so generally accepted that patients with Stage I disease do not require treatment that such cases have been excluded in several controlled trials in sarcoidosis. Thus Hapke's thirty-two patients included only two with thoracic adenopathy alone (1967), and McLean \& Carter's sixteen patients all appear to have had advanced disease (1967). In the study of James, Trowell \& Carstairs (1967) however, twenty-five of fifty-five patients had Stage I disease. It is noteworthy that the trials of Hapke and of McLean \& Carter gave equivocal results while James, Trowell \& Carstairs found significant improvement as the result of treatment. If long-term observation of their Stage I patients shows a continued advantage in the treated group, the combination of their data and ours should provide numbers that are statistically significant.
The suggestion that all patients with Stage I disease should receive treatment with prednisone may appear inappropriate in Europe where most patients presenting with sarcoidosis in this stage clear without treatment. In the United States, however, the prognosis in Stage I is not significantly better than in Stages II and III (Sones \& Israel, 1960; Israel \& Beggs, 1969).

The long-term results in patients with pulmonary disease (Stages II and III) on entry to the trial were unaffected by prednisone. No difference was observed by clinical, radiologic or laboratory criteria at the end of approximately 3.5 years of observation. A 3-months' course of prednisone in daily dosage of $15 \mathrm{mg}$ is clearly insufficient to influence the eventual outcome of pulmonary sarcoidosis. It is of interest that the 6-months' courses employed by Hapke (1969) were no more effective.

The reason for the apparent effectiveness of prednisone in Stage I sarcoidosis and the lack of effect in more advanced disease may be merely quantitative. Patients with Stage I disease have often been shown on lung biopsy to have pulmonary granulomas. The short course of modest doses of prednisone may exert a therapeutic effect on small granulomas but not on larger, roentgenographically demonstrable lesions. Alternatively, the effect in Stage I may be a more fundamental one, reflecting an immunologic difference between disease grossly confined to the mediastinum and disease which has disseminated. It is probable that the primary lesion of sarcoidosis is in the mediastinal lymph nodes; and the hypothesis that treatment at this stage may minimize dissemination and thereby influence the eventual outcome of the disease is a plausible one.

Although corticosteroids exert fairly consistent suppressive and symptomatic effects, patients are encountered in whom prednisone is contraindicated. poorly tolerated, or ineffective. Oxyphenbutazone and chloroquine have been reported in controlled trials to be effective in sarcoidosis but our clinical experience with these agents has been disappointing. Use of oxythenbutazone in many fresh cases has failed to yield symptomatic benefit in any, while chloroquine has proved occasionally useful in patients in whom corticosteroids were poorly tolerated or contraindicated.

More promising in our experience have been chlorambucil and methotrexate. Use of these agents appeared justifiable only in patients whose disease was inadequately controlled by adrenal steroids, or patients by whom corticosteroids could not be tolerated. Fourteen patients have been given chlorambucil, nine methotrexate and six additional patients received both. In half the cases there was symptomatic improvement with little radiologic change; suppression of uveitis was noted in four 
patients. In seven patients, clinical and radiologic improvement was dramatic but relapse occurred 3 to 12 months after cessation of treatment. The rapidity with which improvement occurred, the impermanence of the effects, and the inconstant reduction observed in serum immunoglobulin levels indicate that the effects of these drugs is an antiinflammatory one similar to that of corticosteroids, rather than an immuno-suppressive one. No ill effects were noted from use of these drugs, but they are infrequently more effective than corticosteroids; their use should be limited to occasional cases of progressive sarcoidosis in which corticosteroids are ineffective or contraindicated.

\section{References}

Deenstra, H. \& VAN Ditmars, J. (1968) Sarcoidosis. Diseases of the Chest, 53, 57.

HAPKE, E.J. (1967) Steroid treatment in pulmonary sarcoidosis. La Sarcoidose, Rapports de la IV Conférence internationale, Masson et Cie, Paris, 713-21.

HAPKE, E.J. (1969) Steroid treatment in pulmonary sarcoidosis: A 4-year follow-up of controlled trial. Fifth International Conference on Sarcoidosis, Prague.
Hoyle, C., Smellie, H. \& Leak, D. (1967) Prolonged treatment of pulmonary sarcoidosis with corticosteroids. Thorax, 22, 519.

ISRAEL, H.L. \& BEGGS, R. (1970) A controlled trial of prednisone therapy in sarcoidosis. (In press.)

Israel, H.L., Sones, M. \& Harrell, D. (1954) Cortisone treatment of sarcoidosis: experience with thirty-six cases. Journal of the American Medical Association, 156, 461-6.

James, D.G., Trowell, J.M. \& Carstairs, L.S. (1967) Treatment of sarcoidosis. Controlled therapeutic trial. La Sarcoidose, Rapports de la IV Conférence internationale, pp. 749-54. Masson et Cie, Paris.

MCLean, R.L. \& Carter, F.M. (1967) A controlled trial of corticosteroids in pulmonary sarcoidosis. La Sarcoidose, Rapports de la IV Conférence internationale 0p. 735-41. Masson et Cie, Paris.

SCADDING, J.G. (1967) Sarcoidosis, p. 341. Eyre \& Spottiswoode, London.

Sharma, O.P., Colp, C. \& Williams, M.H. (1966) Course of pulmonary sarcoidosis with and without corticosteroid therapy as determined by pulmonary function studies. American Journal of Medicine, 41, 541.

Siltzbach, L. (1967) Sarcoidosis: Clinical features and management. Medical Clinics of North America, 51, 483.

Sones, M. \& Israel, H.L. (1960) Course and prognosis of sarcoidosis. American Journal of Medicine, 29, 84.

Sones, M., Israel, H.L., Dratman, M.B. \& Frank, J.H. (1951) Effect of cortisone in sarcoidosis. New England Journal of Medicine, 244, 200-13. 